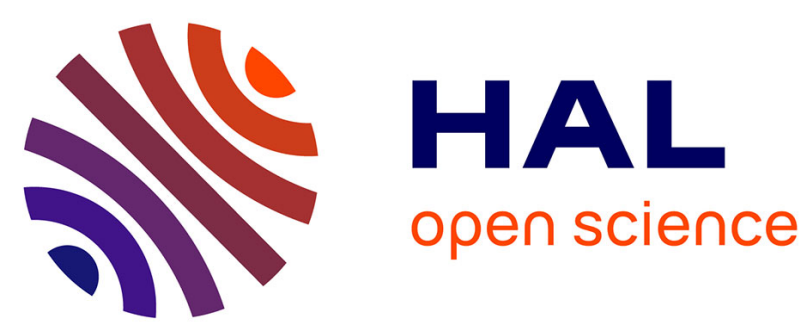

\title{
Enhancement of the photoluminescence property of hybrid structures using single-walled carbon nanotubes/pyramidal porous silicon surface
}

Haythem Gammoudi, Fatma Belkhiria, Kamel Sahlaoui, Walid Zaghdoudi, Mahmoud Daoudi, Saloua Helali, Fabien Morote, Hassan Saadaoui, Mosbah Amlouk, Gediminas Jonusauskas, et al.

\section{To cite this version:}

Haythem Gammoudi, Fatma Belkhiria, Kamel Sahlaoui, Walid Zaghdoudi, Mahmoud Daoudi, et al.. Enhancement of the photoluminescence property of hybrid structures using single-walled carbon nanotubes/pyramidal porous silicon surface. Journal of Alloys and Compounds, 2018, 731 (Supplément C), pp.978 - 984. 10.1016/j.jallcom.2017.10.040 . hal-01629303

\section{HAL Id: hal-01629303 https://hal.science/hal-01629303}

Submitted on 6 Nov 2017

HAL is a multi-disciplinary open access archive for the deposit and dissemination of scientific research documents, whether they are published or not. The documents may come from teaching and research institutions in France or abroad, or from public or private research centers.
L'archive ouverte pluridisciplinaire HAL, est destinée au dépôt et à la diffusion de documents scientifiques de niveau recherche, publiés ou non, émanant des établissements d'enseignement et de recherche français ou étrangers, des laboratoires publics ou privés.

\section{다(1)(2)}

Distributed under a Creative Commons Attribution - ShareAlikel 4.0 International 


\title{
Enhancement of the photoluminescence property of hybrid structures using single-walled carbon nanotubes/pyramidal porous silicon surface
}

\author{
Haythem Gammoudi a, *, Fatma Belkhiria a, Kamel Sahlaoui ${ }^{\text {b }}$, Walid Zaghdoudi ${ }^{\text {c }}$, \\ Mahmoud Daoudi ${ }^{d}$, Saloua Helali ${ }^{a}$, Fabien Morote ${ }^{\mathrm{e}}$, Hassan Saadaoui ${ }^{\mathrm{f}}$, \\ Mosbah Amlouk ${ }^{g}$, Gediminas Jonusauskas ${ }^{\mathrm{e}}$, Touria Cohen-Bouhacina ${ }^{\mathrm{e}}$, \\ Radhouane Chtourou ${ }^{\text {a }}$
}

a LANSER, Laboratoire de nanomatériaux et des systemes pour les énergies renouvelables, Centre de Recherches et des Technologies de l'Energie, Technopole Borj-Cedria, BP.95, Hammam Lif, 2050, Université Tunis el Manar, Tunisia

${ }^{\mathrm{b}}$ LEPT, laboratoire de procedure thermique, Centre de Recherches et des Technologies de l'Energie, Technopole Borj-Cédria, BP.95, Hammam Lif, 2050,

Université Tunis el Manar, Tunisia

${ }^{\mathrm{c}}$ Université de Carthage, Institut Supérieur des Sciences et Technologies de l'Environnement de Borj-Cedria, Technopôle de Borj-Cedria, BP-1003, Hammam Lif, 2050, Tunisia

d Laboratoire de recherche Energie et Matière pour les Développements des Sciences Nucléaire, Centre National des Sciences et Technologie Nucléaires, 2020 Sidi-Thabet, Tunisia

e LOMA, Laboratoire Ondes et Matière d'Aquitaine, Université de Bordeaux CNRS, 351, cours de la Libération, 33405 Talence, France

${ }^{\mathrm{f}}$ Centre de Recherche Paul Pascal - CNRS, Université de Bordeaux, Av. Schweitzer, 33600 Pessac, France

${ }^{\mathrm{g}}$ Unité de physique des dispositifs à semi-conducteurs, Faculté des sciences de Tunis, Université Tunis El Manar, 2092 Tunis, Tunisia

\section{A R T I C L E I N F O}

Keywords:

Single-walled carbon nanotubes

3-Aminopropyltriethoxysilane

Pyramidal porous silicon

Photoluminescence

Raman spectroscopy

\begin{abstract}
A B S T R A C T
This work presents additional physical results about the enhancement of the photoluminescence property of hybrid structures using single walled carbon nanotubes/pyramidal porous silicon surface, in comparison with what has already been published on these structures in terms of synthesis conditions and FTIR investigations as reported recently by the same authors in Journal of Alloys and Compounds 694 (2017) 1036 1044. Herein, the effect of the single walled carbon nanotubes (SWCNTs) layer on the optical properties of pyramidal Porous Silicon (pPSi) in hybrid SWCNTs/pPSi structure synthetized by chemical and electrochemical etching of silicon wafer was studied. Using both scanning electron mi croscopy (SEM), SWCNTs formed a thin film on pPSi surface and they are partly embedded in its pores. An analysis of Raman spectra for the realized structures confirmed the passivation of pPSi surface by SWCNTs film. The surface bond configurations were also monitored. Moreover, SWCNTs modified Pho toluminescence (PL) spectrum of pPSi by shifting PL peaks towards high energies, showed that the defect created in the materials can result in an efficient and stabilized photoluminescence response on Silicon
\end{abstract} (Si).

\section{Introduction}

Carbon nanotubes generally exhibit remarkable physical, chemical and optical properties [1]. They have also interesting electronic properties due to their metallic appearance and semi conductor behavior. Many experimental attempts focused on

\footnotetext{
* Corresponding author. Tel.: +21650884993.

E-mail address: haythemgammoudi@hotmail.fr (H. Gammoudi).
}

hybrid structures widely used in electronic devices [2]. The single walled carbon nanotubes (SWCNTs) were used as an active layer for absorbing light in the molecules [3] and the light trapping in photovoltaic devices [4].

This unique property of quantum defects in SWCNTs, together with their compatibility with Si based nano device fabrication technology opens a new path to realize room temperature single photon sources operating at telecommunication wavelengths that are critically needed for applications in quantum communications.

The pyramidal Porous Silicon (pPSi) seems to be a suitable 
candidate to integrate SWCNTs into the substrate with a large specific surface area. It is prepared by chemical and electrochemical etching of a single crystal with the formation of small cavities, producing a thick wall between the pores whose size may be of several nanometers [5]. In fact, pPSi has some interesting physico chemical properties such as: (i) its intense visible photo luminescence, (ii) its anti reflective properties and (iii) its band gap energy value that increases due to the quantum confined effect in silicon nanocrystals. Due to these characteristics, Si can be used in the production of optoelectronic and photonic devices [6]. Besides, the relatively high chemical and adsorption sensitivity of pPSi large internal surface is actively exploited in the touch sensing elec tronics and biomedical technologies [7].

First attempts to attach SWCNTs in specific surface were done in 2000 by Liu et al. [8] who implanted shortened SWCNTs to gold. Nevertheless, carbon nanotubes were not chemically immobilized to silicon until 2006 when Yu et al. [9] attached shortened SWCNTs to silicon by first hydrogenating a silicon (100) surface. In 2007, they refined chiefly the approach by hydroxylating silicon (100) surfaces and chemically implanting shortened SWCNTs using DCC coupling [10]. In the same period, Flavel et al. [11] obtained a SWCNTs immobilized to an amine terminated silane layer (APTES) on silicon.

By 2010, Cameron et al. [12] reported both the chemical attachment of functionalized SWCNTs to amino silane modified PSi and the formation of silane patterns on PSi. In 2012, the same au thors used PSi functionalized with APTES to immobilize carboxyl ated SWCNTs [13]. Their work showed that there was an optimal concentration of APTES from which there is enough SWCNTs to produce a large amount of current, proving that SWCNTs surface coverage can significantly affect the solar cell performance.

In 2013, the same researchers found that both the chemical attachment and patterning of SWCNTs on PSi show the ability of SWCNTs to capture human neuroblastoma cells [14].

Recently, Gammoudi et al. [15] have modified the silicon topography by a pPSi structure to increase the surface in contact with the carbon nanotube. Then, they have attached chemically SWCNTs on pPSi surface and into the nano pores of pPSi structure. Their observations by means of the Atomic Force Microscopy (AFM) confirmed that the coverage of the attached SWCNTs decreased with decreasing APTES density on the surface.

Creating hybrid structures or composite materials allows mini mizing the size effects and creating a large specific surface area between silicon and SWCNTs [16]. Besides, an interaction between nanoparticles in the hybrid structures can lead to the appearance of new unique properties compared to those of the individual com ponents. In particular, the modulation of fluorescent radiation is detected in SWCNTs/pPSi structures [17]. The pPSi based photo detectors with surface coated by reducing SWCNTs have high sensitivity and quantum efficiency over a wide spectral rangefrom near UV to IR regions [18].

From an overview of the literature, we note that the use of pPSi surface modification with the SWCNT and the effects of SWCNTs on the luminescence of the of pPSi surface in such structures were not been extensively studied [15]. Thus, it would be quite interesting to make physical investigations for the PL properties of this hybrid structure, which could open alternative applications for pPSi as substrates in various optoelectronics and sensitivity devices.

In this work, SWCNTs was used to modify pPSi layers in order to improve its process ability. Due to the high stability of SWCNTs, special attention was focused on the effects of SWCNTs implanta tion on pPSi photoluminescence. Moreover, further physical in vestigations by means of RAMAN and SEM were carried out.

\section{Experimental procedure}

\subsection{Materials}

Single wall carbon nanotubes (SWCNTs) (Fig. 1) containing carboxylic acid functionality were purchased from Sigma Aldrich. The diameter range of SWCNTs was 4-5 nm, the length was be tween 0.5 and $1.5 \mu \mathrm{m}$, and the purity was above 90\%. APTES: 3 aminopropyltriethoxysilane with purity of 99\% (Aldrich) was used to attach functionalized SWCNTs with a carboxylic acid on a porous silicon pyramidal structure (Fig. 1).

\subsection{Substrates preparation}

P type $\left(\begin{array}{lll}1 & 0 & 0\end{array}\right)$ oriented silicon wafers were used in the experi ment. The resistivity of the samples was between 0.01 and $0.02 \Omega \mathrm{cm}$. The surface of samples was of the order of $20 \times 20 \mathrm{~mm}^{2}$. First, pyramidal silicon (pS) structures were formed after chemical etching in $\mathrm{NaOH}(1 \mathrm{M})$ solution at $85^{\circ} \mathrm{C}$ during 6 min to remove $\mathrm{Si}$ damage (Table 1) $[15,19,20]$.

Second, pPSi structure was prepared by electrochemical anod ization of electrolyte solution of $\mathrm{HF}$ (25\%) and EtOH, respectively. A computer controlled current source was used to fabricate porous pyramidal structured silicon from the selected current density and time data, as shown in Table 1. After the electrochemical at tacks, all the samples were rinsed with ethanol and dried in the ambient air to form an oxide layer SiOx [15,21]. After that, APTES was prepared in the mixture with ethanol (Table 1) [15,21]. The sample of pPSi structure was then immerged for $8 \mathrm{~h}$ in the solution of APTES. Later on, it was rinsed with ethanol to remove APTES that was not attached to the surface of porous silicon pyramidal struc ture [15]. Afterwards, a heat treatment to activate pPSi surface with APTES was carried out. This treatment led to the formation of APTES network, which progressively covered the inside of the pores and the entire porous surface. Finally, after being mixed with Dime thylformamide (DMF, $10 \mathrm{ml}$ ) and dispersed via ultra sonication for $30 \mathrm{~min}$, the solution of SWCNTs (Table 1) was deposited applying spray method on pPSi 3 aminopropyltriethoxysilane (pPSi A) sur face [15]. The obtained samples, named pPSi A SWCNTs (pPSi A $\mathrm{CN}$ ), were heated for $1 \mathrm{~h}$ at $150{ }^{\circ} \mathrm{C}$ to dry and tail the disposal.

\subsection{Technical details of the realized structures}

The elaborated structures were studied in detail by both SEM and Atomic Force Microscopy (AFM) in a recent publication by the same authors [15].

SEM images reveal that the properties of pPS layer (porosity, thickness, pore diameter and microstructure) mainly depend on the operating parameters of the layer formation, including the HF concentration ratio, the presence of surface active compound ad ditives, the duration, the temperature as well as the wafer type and its resistivity. The wafer surface was homogeneously covered $(85 \%$ coverage) by pyramidal structure of silicon (pS). The sizes of the pyramids are not uniform and ranging from $2 \mathrm{~mm}$ to $7 \mathrm{~mm}$, with an average pyramid size of $4.5 \mathrm{~mm}$. The facets of the pyramids are all smooth. The obtained porosity was about $80 \%$. The diameters of the pPS pores are identical and their sizes are around $20 \mathrm{~mm}$.

AFM analysis is in accordance with the results obtained by scanning electron microscopy, that confirm the successful im plantation and dispersion of each SWCNTs into the pPS A surface.

\subsection{Characterisation techniques}

The morphology of the samples was characterized using HITA CHI S 4800 scanning electron microscopy (SEM). Raman spectra 


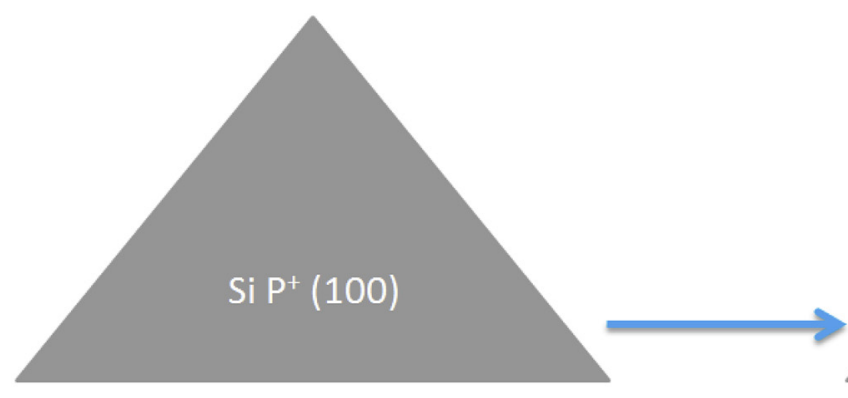

(a): Chemical etching of Si

silicon structure pyramidal: pSi

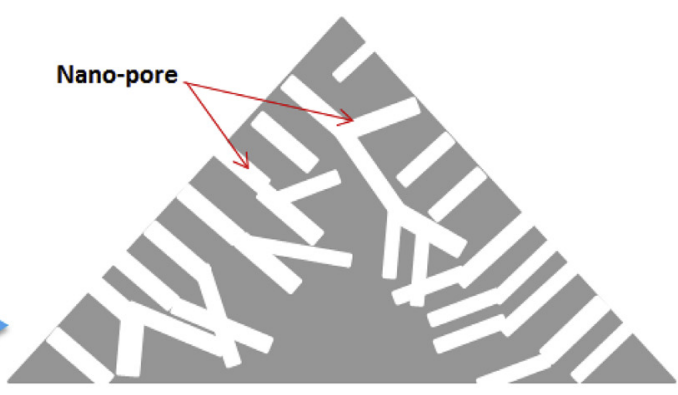

(b): Electrochemical etching of pSi Porous silicon structure pyramidal: pPSi

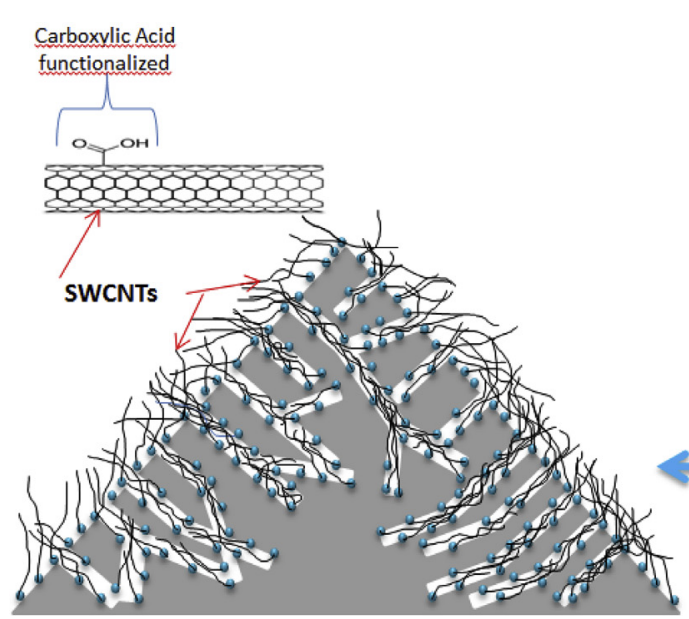

(d): Implantation of SWCNTs Porous silicon structure pyramidal with APTES and SWCNTs : pPSi-A-CN

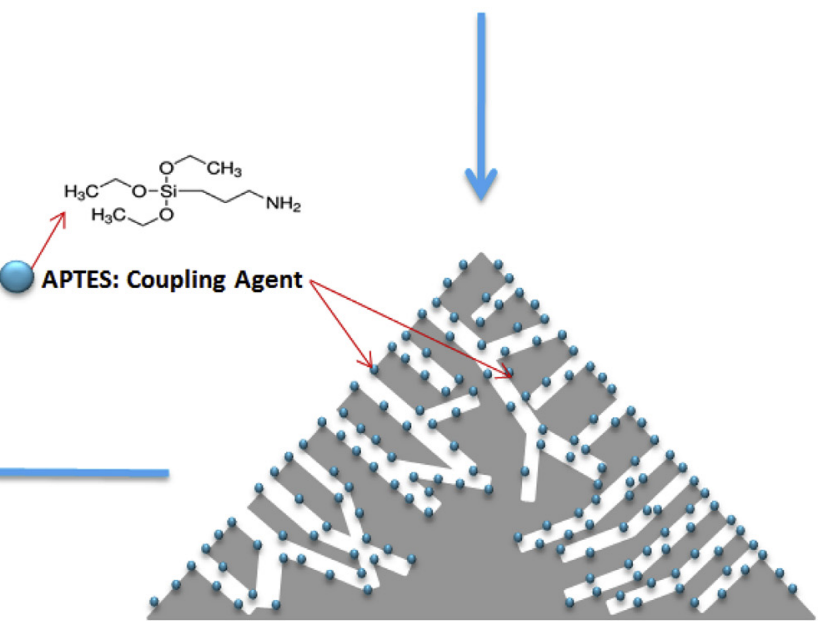

(c): Deposition of APTES Porous silicon structure pyramidal with APTES : pPSi-A

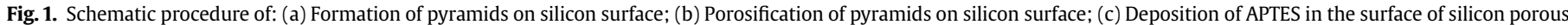
structured pyramidal; (d) Deposition of carbon nanotubes on silicon porous structured pyramidal surface with APTES.

Table 1

Preparation parameters of the porous pyramidal silicon structures and concentration of APTES and SWCNTs.

\begin{tabular}{|c|c|c|c|c|c|}
\hline \multirow[t]{2}{*}{ Samples } & \multirow[t]{2}{*}{ Chemical etching (min) } & \multicolumn{2}{|c|}{ Electrochemical etching } & \multirow[t]{2}{*}{ Concentration of APTES (\%) } & \multirow[t]{2}{*}{ Concentration of SWCNTs (M) } \\
\hline & & Current (mA) & Time $(\mathrm{S})$ & & \\
\hline pS & 6 & & & & \\
\hline pPSi & 6 & 60 & 35 & & \\
\hline pPSi-A & 6 & 60 & 35 & 5 & \\
\hline pPSi-A-CN & 6 & 60 & 35 & 5 & 1 \\
\hline
\end{tabular}

were collected by means of a Raman spectrometer (HORIBA Jobin YvonLabram, HR 800) employing the Argon laser excitation line of $514.5 \mathrm{~nm}$. All spectra were obtained in normal and side view backscattering geometries using a microprobe device that allowed the incident light to be focused on the sample as a spot with a diameter of about $2 \mu \mathrm{m}$. The photoluminescence (PL) mea surements of the prepared structure were performed at room temperature. In PL measurements, the samples were excited with wavelength of $380 \mathrm{~nm}$.

\section{Results and discussion}

\subsection{SEM observations}

Fig. 2 shows the SEM images of the entire structure pPSi A CN. Fig. 2a displays especially the micrograph of pyramidal structure with SWCNTs. The whole wafer surface is covered by pyramidal structure of silicon as shown by Fig. 2a. Furthermore, the sizes of the pyramids are not uniform. The silicon wafers are electro chemically etched to form porous silicon [15]. It is generally assumed that pores initiation occurs at surface active sites defects or irregularities $[12,15]$. More precisely, the image inset with $100 \mathrm{~nm}$ as scale (Fig. 2a) shows the carbon nanotubes which are implanted in the facets of the pyramidal silicon.

\subsection{Raman study}

Raman scattering is a very sensitive technique applied to the microstructure of nano sized materials and to obtain additional information about the properties of all our prepared structures. Fig. 3 depicts the Raman spectra of the realized structure between $100 \mathrm{~cm}^{-1}$ and $3000 \mathrm{~cm}^{-1}$. We observe a peak at $520 \mathrm{~cm}^{-1}$ corres ponding to the silicon in Fig. $3 a$ and $b$ [22]. Another peak appears at $\sim 500 \mathrm{~cm}^{-1}$ from pPSi spectra. It is assigned to porous silicon 


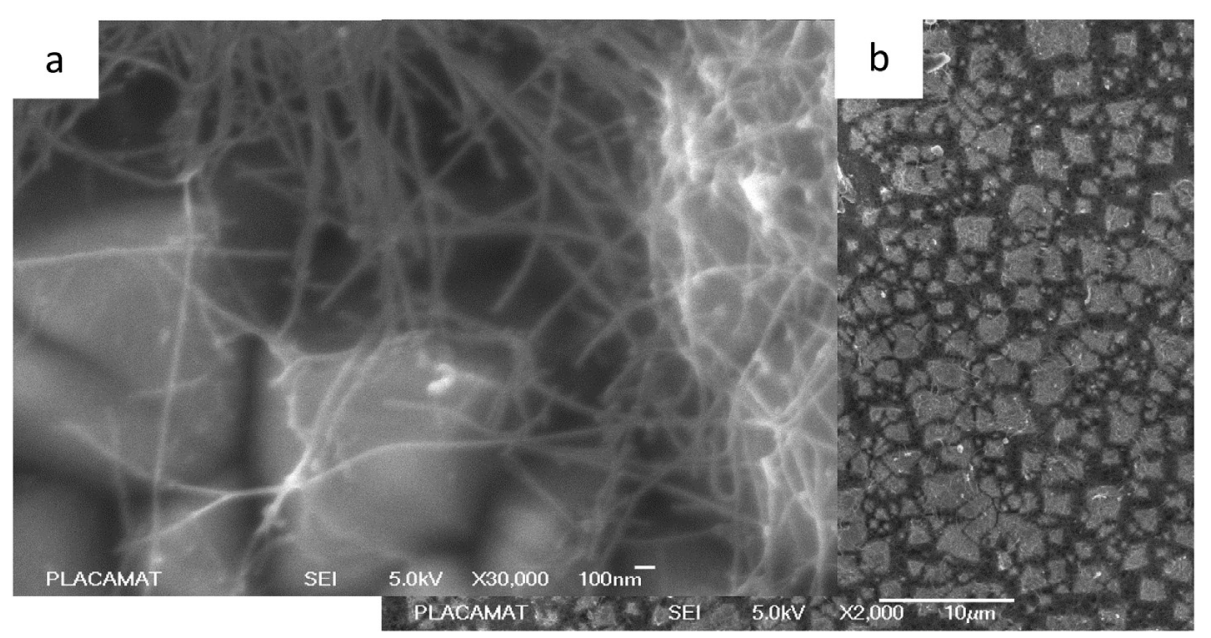

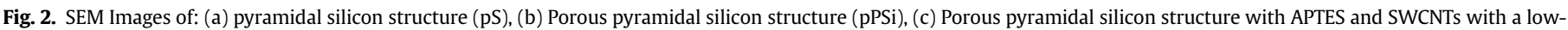
scale view and a large-scale view (pPSi-A-CN).

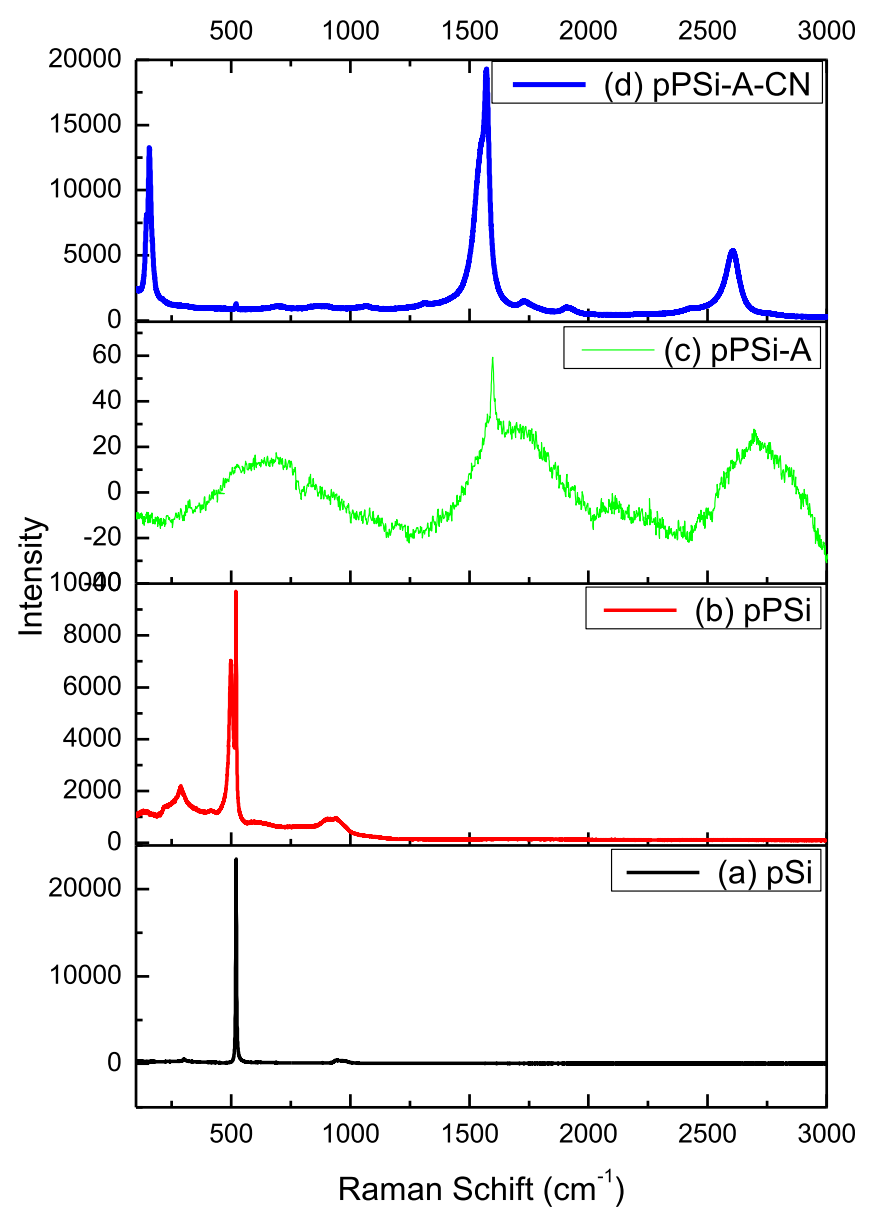

Fig. 3. Raman spectral image of (a) Pyramidal silicon structure pPSi, (b) Pyramidal porous silicon structure (pPSi); (c) Pyramidal porous silicon structure with APTES (pPSi-A), (d) pyramidal porous silicon structure with APTES and SWCNTs (pPSi-A-CN).

(Fig. 3b) [22]. The appearance of peak located at $500 \mathrm{~cm}^{-1}$ (Fig. 3b) is due to the optical phonon scattering at the center of the Brillouin zone of Si [22].

Raman peak intensity is proportional to Si density and photon penetration length in the material [23]. When the porosity increases, these two factors enhance the peak intensity. In fact, if light beams collide with a PS surface, a portion of it scatters from the silicon crystallites and the rest of photons enters in the material and will be scattered by the inner silicon atoms.

Fig. 3b illustrates Raman spectra of functionalized surface with APTES molecules. The bands observed in the spectrum are attrib uted to amine group $\mathrm{NH}_{2}\left(1630 \mathrm{~cm}^{-1}\right)$ [22], $\mathrm{CH}$ stretch modes of $\mathrm{CH}_{2}$ groups of APTES molecules $\left(2700 \mathrm{~cm}^{-1}\right)$ [24] and $\mathrm{NH}_{2}$ bending mode $\left(1600 \mathrm{~cm}^{-1}\right)$ [25]. Indeed, the main characteristic peaks of the silicon modified with APTES are attributable respectively to $v$ ( $\mathrm{Si} O \mathrm{Si}), \mathrm{N}(\mathrm{Si} \mathrm{OH})$ and $\delta(\mathrm{Si} O \mathrm{Si})$. They are assigned as follows: 1064 1047, 963960 and $798792 \mathrm{~cm}^{-1}$. Moreover, the peak assigned to $\nu(\mathrm{Si}-\mathrm{O}-\mathrm{Si})$ presents a shift called "bathochromic effect" between 1064 and $1047 \mathrm{~cm}^{-1}$ (shift of $18 \mathrm{~cm}^{-1}$ for $\mathrm{SiO}_{2}-\mathrm{NH}_{2}$ ). This shift is classically attributed to the structural change of silica after modification. In addition, Raman spectra confirm the presence of bands around $2875 \mathrm{~cm}^{-1}$ for $\mathrm{SiO}_{2}-\mathrm{NH}_{2}$ characteristics for $\mathrm{CH}_{2}$ group. For $\mathrm{SiO}_{2}-\mathrm{NH}_{2}$ modified silica, a peak appears at $2930 \mathrm{~cm}^{-1}$. This peak indicates that the amine is covalently bound to the sur face of the silica particles [26,27].

Fig. 3c depicts Raman spectra of the PPSiA CN sample. The peaks observed in the spectrum are attributed to the radial breathing mode (RBM, $146 \mathrm{~cm}^{-1}$ ), the carbon chain vibration modes $\left(868 \mathrm{~cm}^{-1}\right)$ [28], the stretching vibration of C C $\left(1063 \mathrm{~cm}^{-1}\right)$, the disorder induced mode ( $D$ band, $1337 \mathrm{~cm}^{-1}$ ), the graphitic $\left(E_{2 \mathrm{~g}}\right)$ mode ( $G$ band) split into the $G$ band $\left(1570 \mathrm{~cm}^{-1}\right)$ and the $G+$ band $\left(1586 \mathrm{~cm}^{-1}\right)$, the $\mathrm{M}$ band $\left(1725 \mathrm{~cm}^{-1}\right)$ as well as to the $2 \mathrm{D}$ band $\left(2664 \mathrm{~cm}^{-1}\right)$ [29]. The presence of RBM band in pPSiA CN Raman spectrum confirms the presence of SWCNTs on the surface. The position of the RBM ( $\omega$ RBM) gives information on the diameter of the SWCNTs using the expression: $\omega$ RBM $223.5 / \mathrm{dt}+25.5$ where $\mathrm{dt}$ is the radius of the studied SWCNT. The radius of SWCNTs on pPSi surfaces was calculated at about $1.9 \mathrm{~nm}$, which is in good agree ment with the diameter quoted by the supplier.

We notice the disappearance of the primary amine peaks $\mathrm{NH}_{2}$ and the appearance of a new peak at $1638 \mathrm{~cm}^{-1}$ which are attributed to the $\mathrm{N} \mathrm{H}$ bending of secondary amides [30].

This result is consistent with that obtained using FTIR [15] which shows the presence of silane molecules belonging to APTES attached at the surface of porous silica. 


\subsection{Photoluminescence study}

\subsubsection{Photoluminescence of the realized structure}

The photoluminescence (PL) is one of the significant and powerful studies which allows obtaining more important and precise information on the energy states of impurities and defects. This information is useful in understanding the structural defects in the studied materials. We present, in Fig. 4, the PL spectra of all samples at T $300 \mathrm{~K}$. It can be seen that pSi has no luminescence effect. After electro etchingprocess, a luminescent band appears at $1.9 \mathrm{eV}$ which corresponds to the band gap energy distribution of porous pyramidal silicon [31]. It is found that, after deposition of APTES on pPSi surface, PL spectra essentially governed by two bands are located at $2 \mathrm{eV}$ and at $2.7 \mathrm{eV}$. The band of $2 \mathrm{eV}$ is attributed to porous silicon band gap energy. We notice an intensity increase and a considerable blue shift in energy position relative to pPSi sample. Besides, another band situated at $2.7 \mathrm{eV}$ is attributed to the $\mathrm{Si}$ o Si bond formed after the functionalization of pPS with APTES.

For pPSi A CN sample, SWCNTs addition in the structure shows an increase in PL intensity and a blue shift of porous silicon band. We observe also an additional band treated as two transitions located at 2.629 and $2.765 \mathrm{eV}$.

During the depot preparation step of APTES on the surface of pPSi, the molecules of APTES were attached to the porous pyra midal silicon surface as well as to the inside of the nano pores (Figs. 1c and 3). The reaction between APTES molecules and the porous silicon surface with a controlled oxide layer liberated ethanol with the chemical formula $\mathrm{CH}_{3} \mathrm{CH}_{2} \mathrm{OH}$ (Fig. 5) and pro moted $\mathrm{Si} O \mathrm{Si}$ bond responsible for the increase of the PL signal and the shift to high energies, which can be explained by the quantum confinement in the nano pores of pyramidal porous silicon.

In the same way, Fig. 6 shows the PL spectra of the deposit of APTES on inert Si. Two bands are observed. The first (at $1.9 \mathrm{ev}$ wide) was due to the natural oxidation of Si; whereas the second band (at $2.7 \mathrm{ev}$ ) resulted from the reaction between the APTES on the low layer of neutral oxide [32].

After the deposit step of SWCNTs, we noticed a visible improvement in the PL intensity. As a result, the coating of SWCNT led firstly to the increase of defect of pPSi A structure [31] which favored the quantum confinement [33]. Second, the escape of photons from pPSi A was facilitated by texturing the thin film surface, because the surface scattering phenomenon occurred strongly on the textured surfaces [34]. SWCNT film on pPSi surface

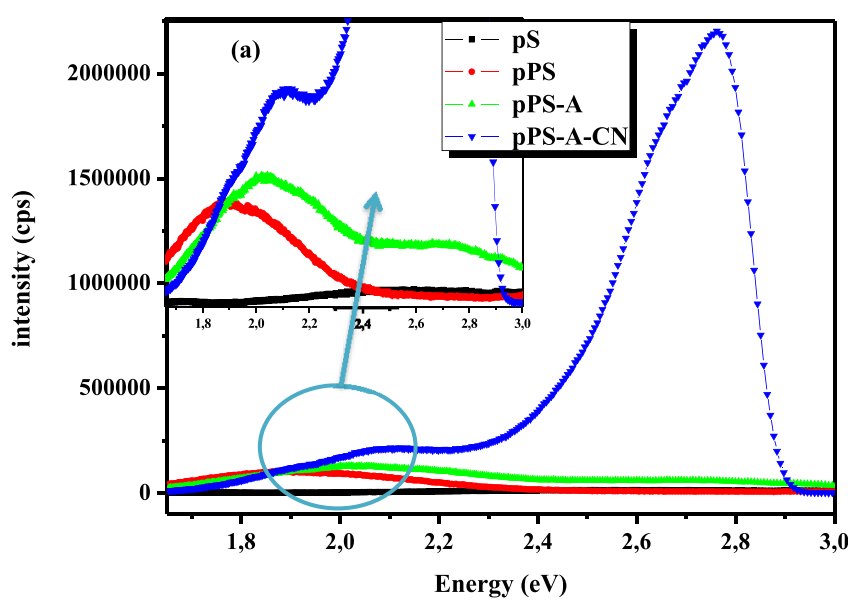

Fig. 4. Photoluminescence spectra of pS, pPSi, pPSi-A and pPSi-A-CN, excited with $380 \mathrm{~nm}$. may lead to such surface scattering phenomenon. On the other hand, the PL band can be associated with the peroxy linkage ((POL, O3Si $O$ O Si O3)) because of the fine structure. We assumed that molecular vibrations influence the PL response [35]. If this light emission originated from $\mathrm{POL}$, the vibration of $\mathrm{O}-\mathrm{O}$ bond would be the most likely source of the fine structure. The defect created in the materials seemed to be involved in the generation of the light emission, which was mainly due to a possible high oxygen content in the sample [36,37]. Therefore, the origin of the PL intensity resulted from the creations of defects in silicon oxide at the SWCNTs/pPSi A interface.

In addition, PL shifts towards high energy region and short wavelength may be ascribed to the recombination of electrons trapped at the states due to Si double bond leading to the quantum confinement effect [38]. In this context, Kanemitsu et al. [39] car ried out PL measurements of the surface oxidized of Si nano crystals. Their results suggested that PL from oxide related inter face states can improve PL response. Mahmoudi et al. [40] observed blue shift of the PL response where CHx modified PS was placed in air without encapsulation which may be understood in terms of the reduction in the size of porous pyramidal silicon resulting in an increase in the silicon energy band gap.

Therefore, we can say that the SWCNTs work as an amplifier of PL intensity.

According to Gammoudi et al. [15], SWCNTs cannot be attached directly to the surface of pSi, or pPSi without the use of APTES as a coupling agent. The authors explored in details the PL measure ments and confirmed the important role of carbon nanotubes in enhancing PL in the realized structure.

\subsubsection{The origin of photoluminescence signal enhancement}

After the activation of the pPSi A layer with SWCNTs, we note a $\mathrm{PL}$ signal enhancement of the structures. For the band at $2 \mathrm{eV}$ the signal increased 1.6 times which states that the substrate is not affected directly with the SWCNTs (The Si $O$ Si was not modified by SWCNTs). For the band at $2.7 \mathrm{eV}$ the enhancement is about 36 times which confirms that the SWCNTs have indeed activated the pPSi A surface and the quantum confinement have occurred.

The improvement in the PL intensity is essentially due to the presence of the SP3 quantum states which favors an increase in the luminescence of the SWCNTs after their dispersion and implanting in the pyramidal porous silicon surface functionalized by APTES Recent studies have shown that such introduction of quantum states is also possible in semiconducting single wall carbon nano tubes (SWCNTs) through low level covalent attachment of various chemical functional groups such as APTES and ether/epoxide $[41,42]$ and a variety of monovalent and divalent alkyl and aryl functionalities [43]. All of these lead to creation of sp3 defects that strongly localize the band edge excitons into quantum states located 100-300 meV [44].

Most defects of SWCNTs are typically known for quenching photoluminescence. However, the quantum states of the $\mathrm{sp} 3 \mathrm{de}$ fects, which we will refer to as quantum defects or defect states, can enhance SWCNT emission efficiency significantly [45].

In fact, the $\mathrm{sp} 3$ quantum states originate from the redistribution of the oxygen defects after the functionalization of pPSi by APTES. Each distribution can be directly associated to the quantum states of the three oxygenated functional groups. This contrast in the spectral behavior provides indications that the attachment of the aryl groups can create a greater number of energetically distinct quantum states [46].

\section{Conclusion}

To conclude, we have successfully synthesized a hybrid 

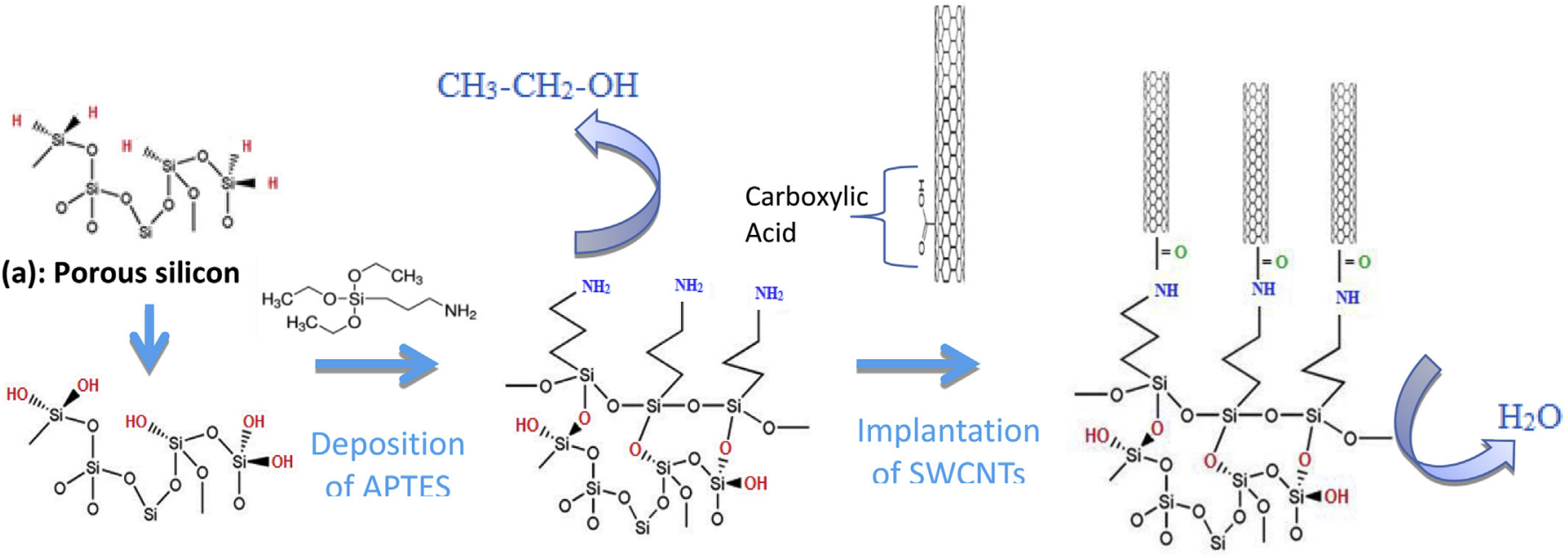

(b): Oxydized Porous Silicon: pPSi

(c): pPSi-A

(d): pPSi-A-CN

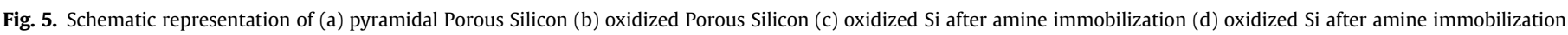
and carboxylic acid attachment.

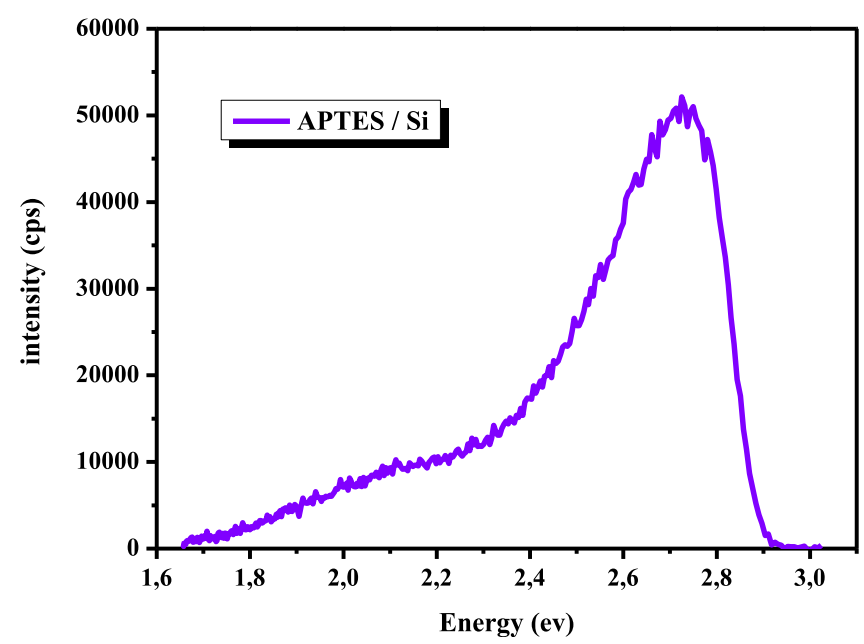

Fig. 6. Photoluminescence spectra of APTES/Si, excited with $380 \mathrm{~nm}$.

structures using single walled carbon nanotube implanted on py ramidal porous silicon surface by means of coupling agent: APTES. All of these individual layers were studied applying the following techniques: SEM, Raman and photoluminescence. It is worth noting that pPSi A CN structures displayed photoluminescent properties in the visible region at room temperature with a noticeably PL response, which may be assigned to the presence of defects from oxygen vacancy. This study seems so interesting and paves the way for further physical investigations to use the method of activation of Si surface with SWCNTs in photovoltaic devices.

\section{Acknowledgment}

The authors gratefully acknowledge all member of LOMA labo ratory, especially, Dr. Christine GRAUBY HEYWANG for their inter esting discussion, Dr. Afrah Bardaoui and Dr. Ibtissem GAMMOUDI for all their advices. We thank Hassan Saadaoui, Cécile ZAKR and all member of Paul Pascal Research Center CRPP for the realization of SEM images. We thank also Marion MATHELIE GUINLET, Zeineb
BEN ABDALLAH, Asma SOUSSOU and Brahim BELGACEM for their encouragement and their scientific support. I thank also my stu dents Najoua BEN AMOR and Insaf BEN ZINA to their encourage ment. I thank finally Pr. Ibrahim BSAIES, Pr Hatem ZAOUIA and Pr Monji BOUAICHA for all their advices. Finally, we thank university of Tunis El Manar and university of Bordeaux for their help.

\section{Nomenclature}

SWCNTs Single Walled Carbon Nanotubes

APTES 3 aminopropyltriethoxysilane

pSi pyramidal Silicon

PSi Porous Silicon

pPSi pyramidal Porous Silicon

pPSi A pyramidal Porous Silicon with APTES layer

pPSi A CN pyramidal Porous Silicon with APTES and SWCNTs layers

SEM Scanning Electron Microscopy

FTIR Fourier transform infrared spectroscopy

UV-Vis UV-visible spectroscopy UV-Vis

PL Photoluminescence

DMF Dimethylformamide

POL Peroxy linkage

\section{References}

[1] R. Saito, G. Dresselhaus, M.S. Dresselhaus, Physical Properties of Carbon Nanotubes, Imperial College Press, London, UK, 1998, p. 11. Ch. 8.

[2] A.A.D.T. Adikaari, D.M.N.M. Dissanayake, S.R.P. Silva, IEEE J. Sel. Top. Quantum Electron. 16 (2010) 1595.

[3] J. Dinitinger, S. Klein, T.W. Ebbesen, Molecule-surface plasmon interactions in hole arrays: enhanced absorption, refractive index changes, and all-optical switching, Adv. Mater. 18 (2006) 12671270

[4] N.M. Jeremy, A.A. Harry, Large integrated absorption enhancement in plasmonic solar cells by combining metallic gratings and antireflection coatings, Nano Lett. 11 (2011) 21952201.

[5] A.G. Cullis, L.T. Canham, P.D.J. Calcott, The structural and luminescence properties of porous silicon, J. Appl. Phys. 82 (1997) 909965.

[6] O. Bisi, S. Ossicini, L. Pavesi, Porous silicon: a quantum sponge structure for silicon based optoelectronics, Surf. Sci. Rep. 38 (2000) 1126.

[7] C. Baratto, G. Faglia, G. Sberveglieri, Z. Gaburro, L. Pancheri, C. Oton, L. Pavesi, Multiparametric porous silicon sensors, Sensors 2 (2002) 121126.

[8] Zhongfan Liu, Ziyong Shen, Tao Zhu, Shifeng Hou, Lizhen Ying, Organizing single-walled carbon nanotubes on gold using a wet chemical self-assembling technique, Langmuir 16 (8) (2000) 35693573. 
[9] Jingxian Yu, Dusan Losic, Matthew Marshall, Till Bocking, John Justin Gooding, Joseph George Shapter, Preparation and characterization of an aligned carbon nanotube array on the silicon (100) surface, Soft Matter 2 (2006) 10811088.

[10] J. Yu, J.G. Shapter, J.S. Quinton, M.R. Johnston, D.A. Beattie, Direct attachment of well-aligned single-walled carbon nanotube architectures to silicon (100) surfaces: a simple approach for device assembly, Phys. Chem. Chem. Phys. 9 (2007) 510520 .

[11] B.S. Flavel, J. Yu, J.G. Shapter, J.S. Quinton, Patterned attachment of carbon nanotubes to silane modified silicon, Carbon 45 (2007) 25512558.

[12] Cameron J. Shearer, Amanda V. Ellis, Joseph G. Shapter, Nicolas H. Voelcker, Chemically grafted carbon nanotube surface coverage gradients, Langmuir 23 (26) (2010) 1846818475

[13] Cameron J. Shearer, Adam Fahy, Matthew Barr, Paul C. Dastoor, Joseph G. Shapter, Improved field emission stability from single-walled carbon nanotubes chemically attached to silicon, Nanoscale Res. Lett. 7 (2012) 432.

[14] Cameron J. Shearer, Frances J. Harding Martin J. Sweetman, Joseph G. Shapter Nicolas H. Voelcker, Nanostructured biointerfaces created from carbon nanotube patterned poroussilicon films, Surf. Coating. Technol. 224 (2013) 4956.

[15] Haythem Gammoudi, Fatma Belkhiria, Saloua Helali, Ibtissem Ben Assaker Ibtissem Gammoudi, Fabien Morote, Ahmed Souissi, Mokhtar Karyaoui, Mosbah Amlouk, Touria Cohen-Bouhacina, Radhouane Chtourou, Chemically grafted of single-walled carbon nanotubes onto a functionalized silicon surface, J. Alloy. Comp. 694 (2017) 10361044.

[16] X. Zhong, G. Wang, B. Papandrea, M. Li, Y. Xu, Y. Chen, C.-Y. Chen, H. Zhou, T. Xue, Y. Li, D. Li, Y. Huang, X. Duan, Reduced graphene oxide/silicon nanowire heterostructures with enhanced photoactivity and superior photoelectrochemical stability, Nano Res 8 (2015) 28502858.

[17] I. Rea, L. Sansone, M. Terracciano, L. De Stefano, P. Dardano, M. Giordano, A. Borriello, M. Casalino, Photoluminescence of graphene oxide infiltrated into mesoporous silicon, J. Phys. Chem. C 118 (2014) 2730127307.

[18] J. Kim, S.S. Joo, K.W. Lee, J.H. Kim, D.H. Shin, S. Kim, S.-H. Choi, Near-ultraviolet-sensitive graphene/porous silicon photodetectors, ACS Appl. Mater. Interfaces 6 (2014) 2088020886

[19] Bohr-Ran Huanga, Ying-Kan Yanga, Wen-Luh Yangb, Key technique for texturing a uniform pyramid structure with a layer of silicon nitride on monocrystalline silicon wafer, Appl. Surf. Sci. 266 (2013) 245249.

[20] Gunjan Aggarwal, Prabhash Mishra, Bipin Joshi, Harsh, S.S. Islam, Porous silicon surface stability: a comparative study of thermal oxidation techniques, J. Porous Mater. 21 (2014) 2329.

[21] M. Terracciano, I. Rea, J. Politi, L. De Stefano, Optical characterization of aminosilane-modified silicon dioxide surface for biosensing, J. Europ. Opt. Soc. Rap. Public 8 (2013) 13075.

[22] K.J. Khajehpour, T. Williams, L. Bourgeois, S. Adeloju, Gold nanothorns macroporous silicon hybrid structure: a simple and ultrasensitive platform for SERS, Chem. Commun. 48 (2012) 53495351.

[23] S. Guha, P. Steiner, F. Kozlowski, W. Lang, Optical characterization of freestanding porous silicon films, J. Porous Mater. 4 (1997) 227237.

[24] Yingying Suna, Masahiro Yanagisawa, Masahiro Kunimoto, Masatoshi Nakamura, Takayuki Homma, Estimated phase transition and melting temperature of APTES self-assembled monolayer using surfaceenhanced anti-stokes and stokes Raman scattering, Appl. Surf. Sci. 363 (2016) 572577.

[25] C.B. Samantaray, J.T. Hastings, Deep UV patterning of 3-amino-propyl-triethoxy-silane self-assembled molecular layers on alumina, J. Vac. Sci. Technol. B: Nanotechnol. Microelectron. Mater. Process.Meas. Phenom 29 (2011) 041603041605

[26] C. Sperandio, A. Laachachi, D. Ruch1, C. Poilâne, P. Bourson, J.-P. Salvestrini, S. Ahzi, Use of functionalized nanosilica to improve thermo-mechanical properties of epoxy adhesive joint bonding aluminium substrates,
J. Nanosci. Nanotechnol. 10 (2010) 28442849.

27] Cameron J. Shearer, Kristina T. Constantopoulos, Nicolas H. Voelcker, Joseph G. Shapter, Amanda V. Ellis, Preparation and characterisation of vertically aligned single-walled carbon nanotube arrays on porous silicon, Smart Mater. V (2008) 726701726711.

[28] Walter Jaimes Salcedo, Francisco J. Ramirez Fernandez, Elisabete Galeazzo, Structural characterization of photoluminescent porous silicon with FTIR spectroscopy, Braz. J. Phys. 27 (1997) 158161.

[29] M.S. Dresselhaus, G. Dresselhaus, R. Saito, A. Jorio, Raman spectroscopy of carbon nanotubes, Phys. Rep. 409 (2005) 4799.

[30] B.H. Stuart, Infrared Spectroscopy: Fundamentals and Applications, WileyVCH, Chichester, England, 2004, pp. 1203.

[31] S. Amdouni, M. Rahmani, M. AZaïbi, M. Oueslati, Enhancement of porous silicon photoluminescence by electroless deposition of nickel, J. Lumin. 157 (2015) 9397.

32] Jing Zhang, Rui Zhang, Li-Hua Zhao, Shu-Oing Sun, Synthesis of water-soluble g-aminopropyl triethoxysilane-capped $\mathrm{ZnO}$ : $\mathrm{MgO}$ nanocrystals with biocompatibility, CrystEngComm 14 (2012) 613619.

[33] Y.J. Fang, J. Sha, Z.L. Wang, Y.T. Wan, W.W. Xia, Y.W. Wang, Behind the change of the photoluminescence property of metal-coated $\mathrm{ZnO}$ nanowire arrays, Appl. Phys. Lett. 98 (2011) 033103.

[34] I. Gontijo, M. Boroditsky, E. Yablonovitch, S. Keller, U.K. Mishra, S.P. DenBaars, Coupling of InGaN quantum-well photoluminescence to silver surface plasmons, Phys. Rev. B 60 (1999) 11564.

[35] T. Trupke, J. Zhao, A. Wang, R. Corkish, M.A. Green, Very efficient light emission from bulk crystalline silicon, Appl. Phys. Lett. 82 (2003) 2996.

[36] Yuryo Sakuraia, Kaya Nagasawab, A study of the PL emission mechanisms in silica glass by considering the growth of the PL, J. Non-Crystalline Solids 291 (1 2) (2001) 8692.

37] Y. Sakurai, K. Nagasawa, Correlation between the $1.5 \mathrm{eV}$ photoluminescencebands and the $3.8 \mathrm{eV}$ absorption band in silica glass, J. Non-Cryst. Solids 261 (2000) 2127.

[38] Y. Sakurai, Correlation between the $1.5 \mathrm{eV}$ photoluminescence band and peroxy linkage in silica glass, J. Non-Cryst. Solids 276 (2000) 159162.

[39] M. Rahmani, A. Moadhen, M.A. Zaibi, H. Elhouichet, M. Oueslati, Photoluminescence enhancement and stabilization of porous silicon passivated by iron, J. Lumin. 128 (2008) 17631766.

[40] Y. Kanemitsu, S. Okamoto, M. Otobe, S. Oda, Photoluminescence mechanism in surface-oxidized silicon nanocrystals, Phys. Rev. B 55 (1997) R7375.

[41] X. Ma, J.K. Baldwin, N.F. Hartmann, S.K. Doorn, H. Htoon, Solid-state approach for fabrication of photostable, oxygen-doped carbon nanotubes, Adv. Funct. Mater. 25 (2015) 61576164.

[42] S. Khasminskaya, F. Pyatkov, K. Słowik, S. Ferrari, O. Kahl, V. Kovalyuk, P. Rath, A. Vetter, F. Hennrich, M.M. Kappes, Fully integrated quantum photonic circui with an electrically driven light source, Nat. Photonics 10 (2016) 727732

[43] H. Kwon, A.O. Furmanchuk, M. Kim, B. Meany, Y. Guo, G.C. Schatz, Y. Wang, Molecularly tunable fluorescent quantum defects, J. Am. Chem. Soc. 138 (2016) 68786885.

[44] H. Kwon, M. Kim, B. Meany, Y. Piao, L.R. Powell, Y. Wang, Optical probing of local $\mathrm{pH}$ and temperature in complex fluids with covalently functionalized semiconducting carbon nanotubes, J. Phys. Chem. C 119 (2015) 37333739.

[45] Y. Miyauchi, M. Iwamura, S. Mouri, T. Kawazoe, M. Ohtsu, K. Matsuda, Brightening of excitons in carbon nanotubes on dimensionality modification, Nat. Photonics 7 (2013) 715719.

[46] xiaowei he, Brendan J. Gifford, Nicolai F. Hartmann, Rachelle Ihly, Xuedan Ma, Svetlana V. Kilina, Yue Luo, Kamran Shayan, Stefan Strauf, Jeffrey L. Blackburn, Sergei Tretiak, Stephen K. Doorn, Han Htoon, Low temperature single carbon nanotube spectroscopy of sp3 quantum defects, ACS Nano 28 (Sep 2017) 140. 